\section{OPEN ACCESS}

Edited by:

Elodie Segura,

Institut Curie, France

Reviewed by:

Aurelie Moreau,

Institut National de la Santé et de la

Recherche Médicale

(INSERM), France

Christophe Chevillard,

INSERM U1090 Technologies

Avancées pour le Génome et la

Clinique, France

${ }^{*}$ Correspondence:

Milena Botelho Pereira Soares

milena@bahia.fiocruz.br

tIn memoriam

Specialty section

This article was submitted to Antigen Presenting Cell Biology,

a section of the journal

Frontiers in Immunology

Received: 19 October 2019

Accepted: 03 March 2020

Published: 07 April 2020

Citation:

Santos ES, Aragão-França LS

Meira CS, Cerqueira $J$,

Vasconcelos JF, Nonaka CKV,

Pontes-de-Carvalho LC and

Soares MBP (2020) Tolerogenic

Dendritic Cells Reduce Cardiac

Inflammation and Fibrosis in Chronic

Chagas Disease.

Front. Immunol. 11:488.

doi: 10.3389/fimmu.2020.00488

\title{
Tolerogenic Dendritic Cells Reduce Cardiac Inflammation and Fibrosis in Chronic Chagas Disease
}

\section{Emanuelle de Souza Santos ${ }^{1}$, Luciana Souza de Aragão-França ${ }^{2}$, Cássio Santana Meira ${ }^{1}$, Jéssica Vieira Cerqueira ${ }^{1}$, Juliana Fraga Vasconcelos ${ }^{1}$, Carolina Kymie Vasques Nonaka ${ }^{1,2}$, Lain Carlos Pontes-de-Carvalho ${ }^{1+}$ and Milena Botelho Pereira Soares ${ }^{1 *}$}

${ }^{1}$ Gonçalo Moniz Institute, FIOCRUZ, Salvador, Brazil, ${ }^{2}$ Center for Biotechnology and Cell Therapy, Hospital São Rafael, Salvador, Brazil

Chronic Chagas disease cardiomyopathy (CCC) is the most frequent and severe form of this parasitic disease. CCC is caused by a progressive inflammation in the heart, resulting in alterations that can culminate in heart failure and death. The use of dendritic cells (DCs) appears as an option for the development of treatments due to their important role in regulating immune responses. Here, we investigated whether tolerogenic cells (tDCs) could interfere with the progression of CCC in an experimental model of Chagas disease. The tDCs were generated and characterized as CD11 $b^{+}$CD11 $c^{+}$cells, low expression of MHC-II, CD86, CD80, and CD40, and increased expression of PD-L. These cells produced low levels of IL-6 and IL-12p70 and higher levels of IL-10, compared to mature DCs (mDCs). Interestingly, tDCs inhibited lymphoproliferation and markedly increased the population of FoxP3 ${ }^{+}$Treg cells in vitro, compared to mature DCs. In a mouse model of CCC, treatment with tDCs reduced heart inflammation and fibrosis. Furthermore, tDCs treatment reduced the gene expression of pro-inflammatory cytokines (Ifng and //12) and of genes related to cardiac remodeling (Col1a2 and Lgals3), while increasing the gene expression of IL-10. Finally, administration of tDCs, increased the percentage of Treg cells in the hearts and spleens of chagasic mice. Ours results show that tolerogenic dendritic cells have therapeutic potential on CCC, inhibiting disease progression.

Keywords: antigen presenting cells, Chagas disease, inflammation, fibrosis, Trypanosoma cruzi, cardiomyopathy

\section{INTRODUCTION}

Chagas disease is caused by the protozoan parasite Trypanosoma cruzi, and affects about 8 million people worldwide, mainly low income populations (1). Although considered a neglected tropical disease endemic in Latin America, this disease is spreading to other continents, such as Europe, North America, Asia and Oceania, due to human migration $(2,3)$. The acute phase of Chagas disease is characterized by high parasitemia with immune-inflammatory response to the parasite, which result in organ damage (4). After the initial infection, most of the patients remain asymptomatic without clinical manifestations. About $20-40 \%$ of the chronic patients, however, develop the cardiac and/or digestive forms of the disease, for which there are no available treatments $(5,6)$.

The chronic Chagas disease cardiomyopathy (CCC) is most frequent and severe manifestation found, with symptoms that range from mild to severe cardiac remodeling associated with 
inflammation, fibrosis, arrythmias and thromboembolic events, which may lead to congestive heart failure and sudden death $(7,8)$. In addition, microvascular ischemia is directly involved in the histopathological pattern of CCC, which consists of a diffuse focal myocarditis associated with myocytolysis and fibrosis (5).

The only available treatment for end-stage CCC patients is heart transplantation, a high-cost procedure not available in time for all patients. Furthermore, organ transplantation presents several complications in patients with chronic infection due to immunosuppressive therapy, which may trigger reactivation of infection $(4,7,9)$. Alternative treatments are needed, with the aim of reducing morbidity and mortality, and providing a better quality of life for the patients with CCC. Therapeutic alternatives focused on reducing the immunological mechanisms, which are key factors in the pathogenesis of the disease, modulating both inflammation and fibrosis in the heart of the patients, are of great interest (10-16).

In this context, immunotherapy with dendritic cells (DCs) appears as an option for the development of treatments based on their role in immune responses. Dendritic cells are APCs which can activate $\mathrm{T}$ cells and induce $\mathrm{T}$ cell tolerance (15). They are important as mediators for the induction of effective immunity against invading pathogens, by connection of innate and adaptive immune responses and maintenance of immune tolerance $(17,18)$. Dendritic cells (DCs) represent a heterogeneous population of potent APCs that include multiple subsets which different functional specializations that depends to their origin, maturation state, location, and environmental conditions (18, 19). The role of DCs in induction of immunity or tolerance is determined by their maturation state (17). Mature DCs mediate immune responses under inflammatory conditions, whereas immunological tolerance is induced by DCs with regulatory profile, characterized by an immature phenotype (19), with low expression of co-stimulatory and MHC molecules, distinct cytokine profile and expression of inhibitory molecules (18).

Considering the role of DCs in both the induction of adaptative immunity and in maintaining immunological tolerance, much attention has been given to the role of these cells in stimulating or preventing autoimmune diseases, associating the potential application of DCs as regulators for treatments of autoimmune diseases. Thus, tolerogenic dendritic cells (tDCs) are a promising therapeutic tool to reduce or prevent autoimmune diseases, including systemic lupus erythematosus (20), atherosclerosis (21), asthma allergic (22), rheumatoid, and inflammatory arthritis (23), experimental autoimmune myocarditis (24), myocardial infarction (25), immunotherapy of cancer (26), and solid organ transplantation (19).

In the present study, we aimed to investigate whether tolerogenic dendritic cells can modulate the inflammation in a mouse model of CCC. We show here that immunotherapy with tolerogenic dendritic cells are able to ameliorate heart inflammation and fibrosis, two hallmarks of CCC.

\section{MATERIALS AND METHODS}

\section{Animals and Trypanosoma cruzi Infection}

Female C57BL/6 mice were maintained in the animal facility of the Center for Biotechnology and Cell Therapy, Hospital São Rafael (Salvador, Bahia, Brazil), and provided with rodent diet and water ad libitum. Trypomastigotes of the myotropic Colombian T. cruzi strain were obtained from culture supernatants of infected LLC-MK2 cells. Infection of C57BL/6 mice was performed by intraperitoneal (i.p.) injection of 1000 T. cruzi trypomastigotes in saline, and parasitemia was monitored during acute infection. All experiments were carried out in accordance with the recommendations of Ethical Issues Guidelines and were approved by the local ethics committee for animal use under number 017/2017.

\section{Generation of Dendritic Cells}

The protocol used to produce dendritic cells (DCs) was adapted from a previously described methodology (22). Bone marrow from $\mathrm{C} 57 \mathrm{BL} / 6$ mice was collected by flushing the femurs with RPMI medium (Sigma-Aldrich). The cells were then cultured in $75 \mathrm{~cm}^{2}$ flasks at a concentration of $10^{6}$ cells/mL in RPMI medium supplemented with $100 \mathrm{mM}$ pyruvate, $200 \mathrm{mM}$ glutamine, $10 \mathrm{mM}$ HEPES, 10\% fetal bovine serum (FBS; GIBCO), $50 \mu \mathrm{g} / \mathrm{mL}$ gentamicin, $0.2 \% \mathrm{NaHCO}_{3}$, and $30 \%$ culture supernatant of X-63 cell line (which produces GM-CSF), at $37^{\circ} \mathrm{C}$ in a $5 \% \mathrm{CO}_{2}$ atmosphere. To generate tolerogenic dendritic cells (tDCs), dexamethasone $\left(10^{-6} \mathrm{M}\right.$; Prodome Laboratory, Campinas, Brazil) was added to the medium at the third day of culture. On day 7 , tDCs were activated with $1 \mu \mathrm{g} / \mathrm{mL}$ of Escherichia coli lipopolysacharide (LPS; Sigma-Aldrich) for $24 \mathrm{~h}$. Control DCs (mDCs) were generated in the same conditions, with the exception of addition of dexamethasone on the cultures.

\section{Characterization of Dendritic Cells}

For immunophenotyping, activated DCs or tolerogenic DCs were incubated with monoclonal antibody ( $\mathrm{mAb}$ )- fluorochrome or biotin conjugates: anti-CCR7-PerCP, anti-CD11c-FITC, antiCD11b-PE, anti-CD40-PE, anti-CD80-PE, anti-CD86-PE, antiMHC-II-biotin, and anti-PD-L1-biotin (eBioscience Inc.; San Jose, CA) or with the corresponding isotype controls for $20 \mathrm{~min}$ at $4{ }^{\circ} \mathrm{C}$ in the dark and washed twice with saline solution with $1 \%$ FBS. PE-avidin was added to the cell suspensions previously incubated with biotin-mAb conjugates, for $20 \mathrm{~min}$ at $4^{\circ} \mathrm{C}$ in the dark, followed by washing once with $1 \%$ FBS saline solution. For each sample, data from 100,000 cells was acquired by three-color flow cytometry, using a BD LSRFortessa SORP cytometer and a BD FacsDiva v.6.2 software (Becton Dickinson; Heidelberg, Germany). Cell-free supernatants of $\mathrm{mDCs}$ or tDCs were collected $24 \mathrm{~h}$ after stimulation and stocked at $-20^{\circ} \mathrm{C}$ until used for cytokine measurements. The concentrations of IL-6, IL10 and IL-12 cytokines were measured by ELISA, using specific antibody kits (R\&D Systems, Minneapolis, MN), according to manufacturer's instructions.

\section{Lymphoproliferation Assay}

For lymphoproliferation assay, splenocyte suspensions from infected C57BL/6 mice (3 months post-infection) were prepared 
in RPMI medium (Sigma-Aldrich) supplemented with $100 \mathrm{mM}$ pyruvate, $200 \mathrm{mM}$ glutamine, $10 \mathrm{mM}$ HEPES, 10\% FBS, $50 \mu \mathrm{g} / \mathrm{mL}$ gentamicin and $0.2 \% \mathrm{NaHCO}_{3}$. Splenocytes $\left(10^{5}\right.$ cells $/ \mathrm{mL}$ ) were plated in 96 well plates, in quadruplicate, in a final volume of $200 \mu \mathrm{L}$, in presence of $2 \mu \mathrm{g} / \mathrm{mL}$ concanavalin A (Con A; Sigma-Aldrich). The mDCs or tDCs were added at 1:10 ratio. After $48 \mathrm{~h}$, cultures were pulsed with $1 \mu \mathrm{Ci}$ of methyl3H-thymidine (Perkin Elmer, Waltham, MA) and incubated for additional $18 \mathrm{~h}$. The cells were then harvested and the ${ }^{3} \mathrm{H}$-thymidine uptake was determined using a $\beta$-plate counter (Multilabel Reader, Finland). An aliquot of cell-free supernatants was collected $24 \mathrm{~h}$ after incubation of splenocytes plus mDCs or tDCs for cytokine measurement. Concentrations of IL-2 and IFN $\gamma$ cytokines were measured by ELISA, using specific antibody kits (R\&D Systems), according to manufacturer's instructions.

\section{CFSE Staining}

Splenocytes from infected C57BL/6 (3 months post-infection) mice were plated into 24 -well plates at a cell density of $10^{5}$ cells $/ \mathrm{mL}$ in RPMI medium supplemented with $10 \%$ FBS containing $2 \mu \mathrm{g} / \mathrm{mL}$ of Con $\mathrm{A}$ in the absence or presence of $\mathrm{mDC}$ or $\mathrm{tDCs}$ (1:10 ratio) for $72 \mathrm{~h}$. Quantitative evaluation of the exponential cell expansion was estimated by the carboxyfluorescein succinimidyl ester-CFSE assay (Invitrogen/Molecular Probes). CFSE staining was performed according to methodology previously described (27). Before acquisition, cells were centrifuged and the pellet was washed twice with cold PBS and labeled with APC anti-mouse CD3 (Biolegend, San Diego, CA) diluted 1:100 for $15 \mathrm{~min}$. Acquisition was performed using a BD LSRFortessa SORP cytometer and data were analyzed using FlowJo software (Tree Star, Ashland, OR). A total of 100,000 events were acquired.

\section{T Regulatory Cells Quantification}

For quantification of $\mathrm{T}$ regulatory cells, splenocytes cultured with Con A $(2 \mu \mathrm{g} / \mathrm{mL})$ in the absence or presence of $\mathrm{mDCs}$ or tDCs were stained to $\mathrm{CD} 4{ }^{+} \mathrm{CD} 25^{+}$Foxp $3^{+}$Treg cells using the Mouse Regulatory T Cell Staining Kit (eBioscience), according to the manufacturer's recommendations. Briefly, $10^{6}$ splenocytes were stained with anti-CD4-FITC and anti-CD25-APC, followed by permeabilization with cold Fix/Perm Buffer and blocking with anti-mouse CD16/32. Then, anti-Foxp3-PE (eBioscience) was added for intracellular Foxp3-staining. Labeled cells were analyzed using a LSRFortessa cytometer and FlowJo software.

\section{Immunotherapy With tDCs}

Groups of 10 chronic chagasic mice (3 months post-infection) were treated with four monthly intraperitoneal injections of $5 \times 10^{5} \mathrm{tDCs}$, or equal volume of saline $(100 \mu \mathrm{L})$. The DC were generated independently for each intraperitoneal injection. Two weeks after the last injection, mice were euthanized under anesthesia with $5 \%$ ketamine (Vetanarcol ${ }^{\circledR}$; Konig, Avellaneda, Argentina) and $2 \%$ xylazine (Sedomin ${ }^{\circledR}$; Konig). Hearts were collected and divided into two halves, being one frozen at $-80^{\circ} \mathrm{C}$ and the other fixed in $10 \%$ buffered formalin, for PCR and histopathology, respectively. Spleens were frozen in
Tissue-Tek (Sakaru, Alphen a den Rijn, The Netherlands), for immunofluorescence analysis.

\section{Histology and Morphometric Analyses}

Heart sections were analyzed by light microscopy after paraffin embedding, followed by standard hematoxylin and eosin (H\&E), and Sirius red staining methods for evaluation of inflammation and fibrosis, respectively, by optical microscopy. Images were digitized using a color digital video camera (CoolSnap, Montreal, Canada) adapted to a Bx41 microscope (Olympus, Tokyo, Japan). Morphometric analyses were performed using the software Image Pro Plus v.7.0 (Media Cybernetics, San Diego, CA). The inflammatory cells were counted in 10 randomly captured fields (x400 view). The percentage of fibrosis was determined using Sirius red-stained heart sections and Image-Pro Plus v.7.0 to integrate the areas, 10 fields per animal were randomly captured using x200 view. Analyses were performed in a blinded fashion.

\section{Real-Time Reverse Transcription Polymerase Chain Reaction (RT-qPCR)}

RNA was extracted of the heart samples using TRIzol (Invitrogen-Molecular Probes, Eugene, OR). The cDNA was synthetized using the High Capacity cDNA Reverse Transcription KIT (Applied Biosystems, Foster City, CA). The qPCR was prepared with TaqMan ${ }^{\circledR}$ Universal PCR Master Mix (Applied Biosystems). qRT-PCR assays were performed to detect the expression levels of Il12 (Mm_00434165_m1), Col1a2 (Mm_00483888_m1), Lgals3 (Mm_00802901_m1), Il10 (Mm_00439616_m1), Ifng (Mm_00801778_m1), and Foxp3 (Mm_00475162_m1). All reactions were run in triplicate on an ABI 7500 Real Time PCR System (Applied Biosystems) under standard thermal cycling conditions. A non-template control (NTC) and non-reverse transcription controls (No-RT) were also included. The samples were normalized with Hprt (Mm_00484683_m1). The threshold cycle (2- $\Delta \Delta \mathrm{Ct})$ method of comparative PCR was used to analyze the data (28).

\section{Immunofluorescence Analysis}

Sections of formalin-fixed paraffin-embedded hearts and frozen spleens were used for detection of CD3 and Foxp3 expression by immunofluorescence. First, paraffin-embedded sections were deparaffinized and submitted to a heat-induced antigen retrieval step by incubation in citrate buffer $(\mathrm{pH}$ 6.0). Then, sections were incubated overnight with the following primary antibodies: anti-CD3 (1:400; BD Biosciences) and anti-Foxp3 (1:400; Dako, Glostrup, Denmark). On the following day, secondary antibodies anti-goat IgG Alexa Fluor 488 (1:600; Molecular Probes) or antirabbit IgG Alexa Fluor 568 conjugated (1:100; Molecular Probes), diluted in $1 \%$ BSA in PBS, were added. Nuclei were stained with 4,6-diamidino-2-phenylindole (DAPI; VectaShield Hard Set mounting medium with DAPI H-1500; Vector Laboratories, Burlingame, CA). Images were analyzed using a confocal laser scanning microscope A1R (Nikon, Tokyo, Japan) and Image-Pro Plus version 7.01 (Media Cybernetics, Rockville, $\mathrm{MD})$. Quantifications of $\mathrm{CD}^{+} / \mathrm{Foxp}^{+}$cells percental area were performed in 10 fields randomly captured under $\mathrm{x} 400$ magnification, using Image-Pro Plus v.7.0. 


\section{Statistical Analyses}

The normality of the data was determined by the Shapiro-Wilk normality test. In order to analyze differences among groups, the one-way analysis of variance test followed by the Newman Keuls test was used for parametric data and the Kruskal-Wallis test followed by Dunn's post-test was used for nonparametric data. To compare the means of the two groups, Mann-Whitney's $U$ test for nonparametric data and Student's $t$ test for parametric data were used. All analyses were performed using Prism version 5.01 (GraphPad Software, San Diego, CA). All differences were considered significant at values of $p<0.05$.

\section{RESULTS}

\section{Characterization of Tolerogenic Dendritic Cells (tDCs)}

Initially, we determined the phenotype of the tDCs generated in the presence of dexamethasone for induction of tolerogenic profile (Figure 1). The analysis by flow cytometry of $\mathrm{CD}_{11} \mathrm{~b}^{+}$ $\mathrm{CD} 11 \mathrm{c}^{+}$population showed a lower expression of CD40, CD80, CD86, and MHC-II in tDCs (Figures 1A-C). This was confirmed by significantly loss in the mean intensity fluorescent $(\Delta \mathrm{MFI})$ of these markers in tDCs (Figure 1D). In contrast an increase expression of CCR7 and PD-L1, not accompanied by a significant increase in $\triangle \mathrm{MFI}$, was observed in cultures of $\mathrm{tDCs}$ compared to mDCs (Figures 1B-D). Additionally, the tDCs produced lower levels of IL-6 and IL-12, and higher levels of IL-10, compared to $\mathrm{mDCs}$ (Figures $\mathbf{2 A}-\mathbf{C}$ ). These results indicate a regulatory phenotype of tDCs generated from bone marrow cells by treatment with dexametosone in vitro.

\section{Modulation of Cell Proliferation in vitro by tDCs}

To demonstrate a modulatory effect of tDCs in lymphocyte activation, a lymphoproliferation assay was performed using splenocytes from $T$. cruzi-infected mice stimulated with concanavalin A. As shown in Figure 3A, the tDCs caused a significant inhibition of lymphoproliferation, while the addition of mDCs did not cause a significant inhibition. Similarly, by using CFSE staining, a marker of cell proliferation, we found that tDCs, but not mDCs, are able to inhibit Con A-induced lymphoproliferation (Figure 3E). Interestingly, addition of tDCs also increased the population of FoxP3 ${ }^{+}$Treg cells, compared to the control mDCs (Figure 3B). Concomitantly, tDCs, but not $\mathrm{mDCs}$, caused a significant reduction in IL- 2 and IFN- $\gamma$ cytokine production (Figures 3C,D).

\section{Immunotherapy With Tolerogenic Dendritic Cells Prevents Progression of CCC}

The therapeutic effects of tDCs was then evaluated in a mouse model of CCC. Heart sections were examined after stained with hematoxylin and eosin and Sirius red for quantification of inflammation and fibrosis, respectively. A multifocal inflammation, mainly composed by mononuclear cells, was found in the hearts of saline-treated T. cruziinfected mice compared to naïve mice (Figures 4A,B). The administration of tDCs led to a significantly reduction in the number of inflammatory cells compared to saline-treated mice (Figures 4C,D).

Mice chronically infected with $T$. cruzi had an increased production of inflammatory mediators in the heart, compared to naïve mice (Figure 4). In the hearts of tDCs treated mice, however, the analysis of gene expression showed a reduction of the pro-inflammatory cytokines IL-12 and IFN- $\gamma$, while IL-10 expression was increased, when compared to vehicle-treated mice (Figures 4E-G).

Similarly, infected mice presented a higher percentage of fibrosis in the heart when compared to naïve controls (Figures 5A,B). Treatment with tolerogenic dendritic cells also promoted a reduction in the percentage of fibrosis when compared with saline-treated mice (Figures 5C,D). This was correlated with a reduction on gene expression of collagen $1 \mathrm{a} 2$ and galectin 3, two factors related to fibrogenesis, as shown by RT-qPCR (Figures 5E,F).

\section{Administration of Tolerogenic Dendritic Cells Increases the Percentage of Treg Cells in the Hearts and Spleens of Chagasic Mice}

One of the effector mechanisms by which tolerogenic dendritic cells act is the induction of Treg cells (24). We next evaluated whether the administration of tolerogenic dendritic cells induces the increase of Treg cells in $T$. cruzi-infected mice. FoxP3 expression was investigated in $\mathrm{CD}^{+}$cells in spleen sections, by immunofluorescence. The percentage of $\mathrm{CD}^{+}$FoxP3 $^{+}$Treg cells increased markedly in the spleens of mice treated with tolerogenic dendritic cells, compared with the group saline-treated mice (Figures 6A-D). Concomitantly, an increase in the level of FoxP3 mRNA was observed in the hearts of tolerogenic dendritic cells-treated mice, compared with that of the group treated with saline (Figure 6E).

\section{DISCUSSION}

CCC is characterized by persistent inflammation leading to extensive cardiac, with progressive damage to the myocardium and heart dysfunction $(29,30)$. In the present study, the use of tolerogenic dendritic cells as a therapeutic tool for CCC was examined using mouse model. Our results show that administration of tolerogenic dendritic cells promoted reduction of heart inflammation and fibrosis, two important features of Chagas heart disease.

We generated tolerogenic dendritic cells in the presence of GM-CSF by treating in vitro with dexamethasone, followed by LPS-induced maturation. The multifunctional cytokine GM-CSF modulates the growth and function of most leukocytes and plays a key role in mediating the differentiation and growth of DCs from mononuclear cells (31). The use of dexamethasone as an agent for the induction of tolerogenic profile in DCs has been previously shown in both mouse bone marrow cells and from human monocytes (32-34). Tolerogenic dendritic 
A

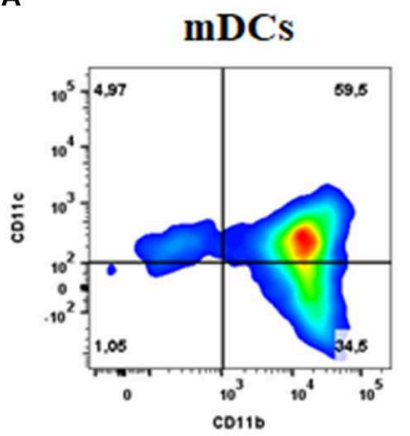

tDCs

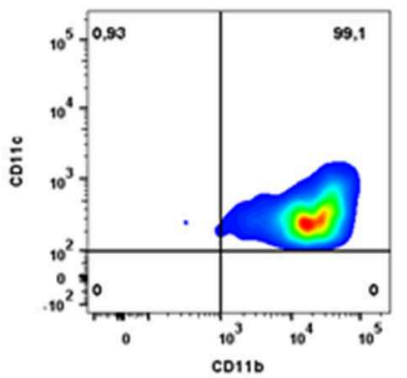

B

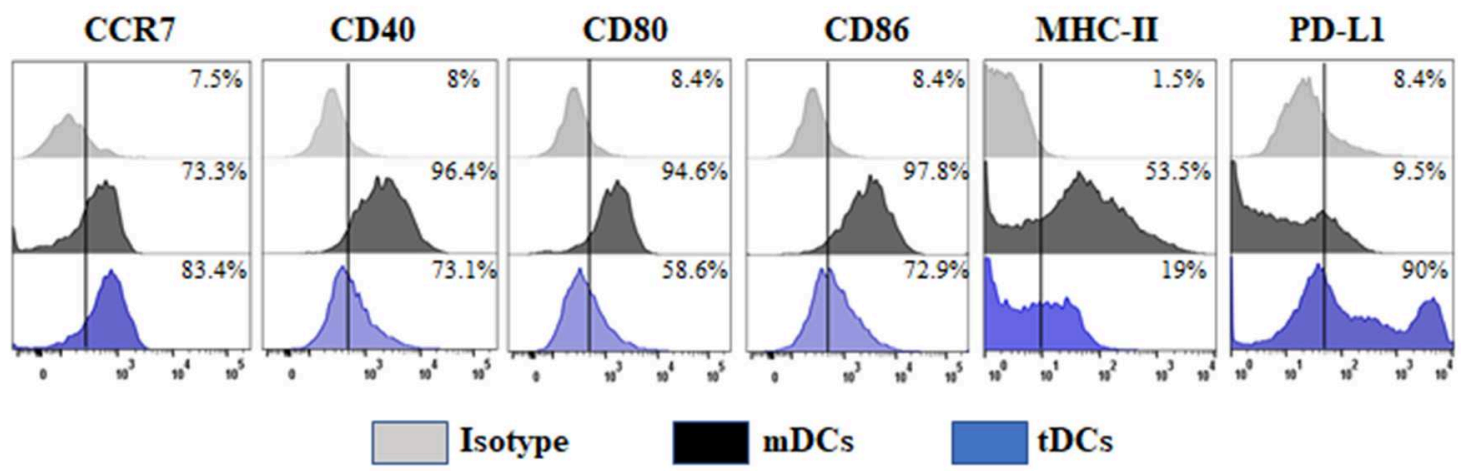

C

D
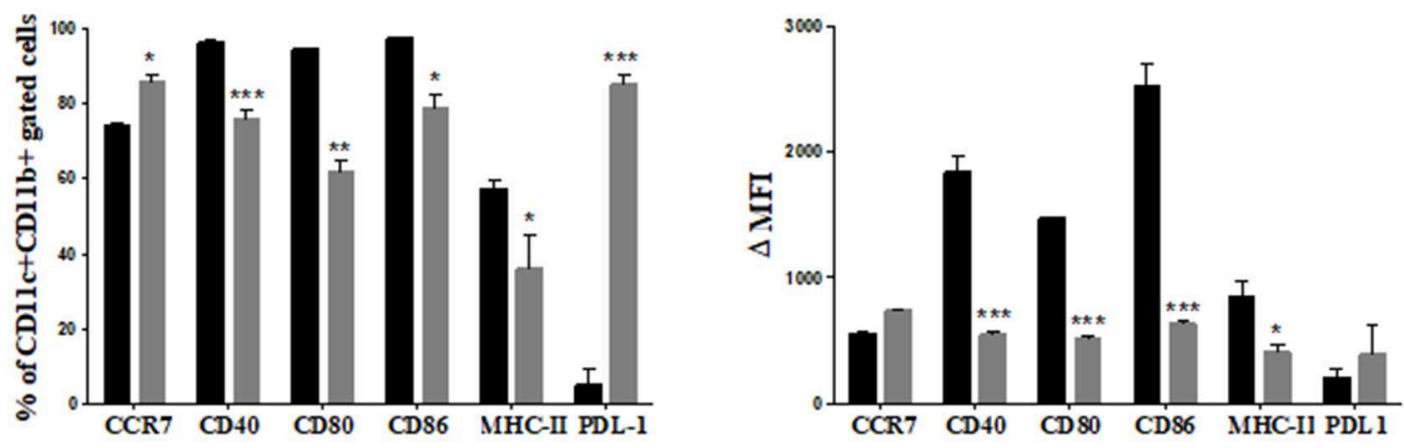

mDCs

tDCs

FIGURE 1 | Immunophenotyping of differentiated DCs from bone marrow cells in the presence of dexamethasone. (A) Dot plot representing the percentage of $\mathrm{CD}_{11 \mathrm{c}^{+}} \mathrm{CD} 11 \mathrm{~b}^{+}$cells. (B) Histograms showing the expression of CCR7, CD40, CD80, CD86, MHC-II, and PD-L1 in mDCs (black) and tDCs cells (blue), within the

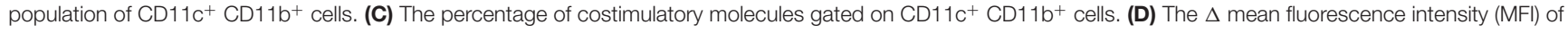
costimulatory molecules gated on $\mathrm{CD} 11 \mathrm{c}^{+} \mathrm{CD}_{11 \mathrm{~b}^{+}}$cells. MHC, major histocompatibility complex; PD-L, programmed death ligand. Values represent the mean of nine determinations \pm SEM of three experiments performed. ${ }^{\star \star \star} P<0.001$; compared to $\mathrm{mDC}$ cells. ${ }^{\star \star} P<0.01$; compared to mDC cells. ${ }^{\star} P<0.05$; compared to $\mathrm{mDC}$ cells.

cells derived by the use of pharmacological agents, such as dexamethasone alone or in combination, and subsequently induced maturation with LPS has previously been shown to acquire stable semi-mature and tolerogenic phenotype (35-38). Furthermore, the use of LPS to generate tolerogenic dendritic cells has proved to be important to favor the regulatory and migratory capacity of these cells (34). In our study, this protocol generated tDCs with low expression of CD40, CD80, CD86, and MHC-II costimulatory molecules, and low production of the proinflammatory cytokines IL-6 and IL-12, 

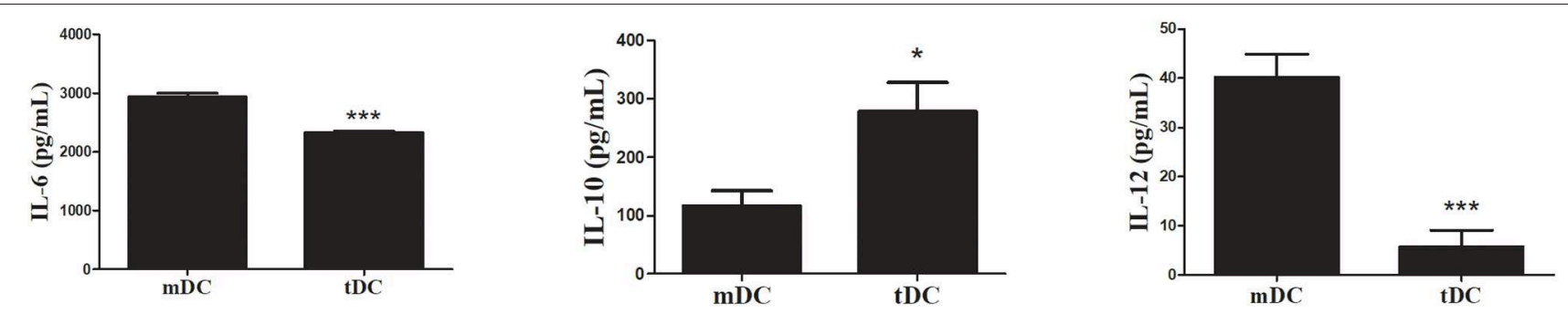

FIGURE 2 | TDCs exhibit an anti-inflammatory cytokine secretion profile. Concentrations of IL-6 (A), IL-10 (B), and IL-12p70 (C) were determined in mDCs or tDCs supernatants by ELISA. Values represent the mean of nine determinations $\pm \mathrm{SEM}$ of three experiments performed. ${ }^{\star \star \star} P<0.001 ;$ compared to $\mathrm{mDC}$ cells. ${ }^{\star} P<0.05$; compared to $\mathrm{mDC}$ cells.
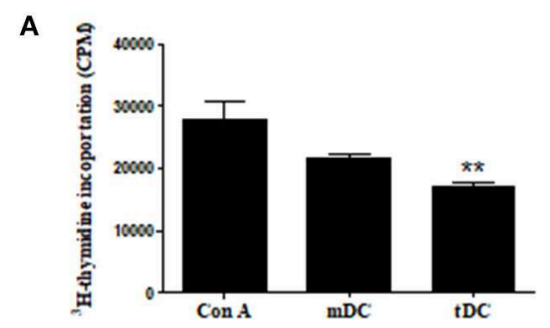

$\mathbf{C}$

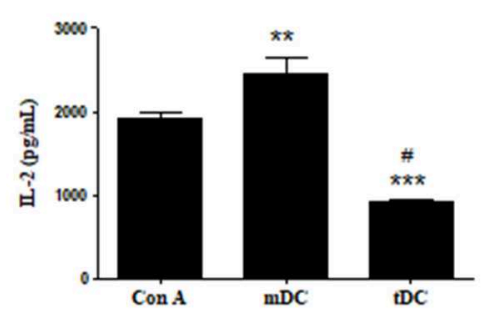

E

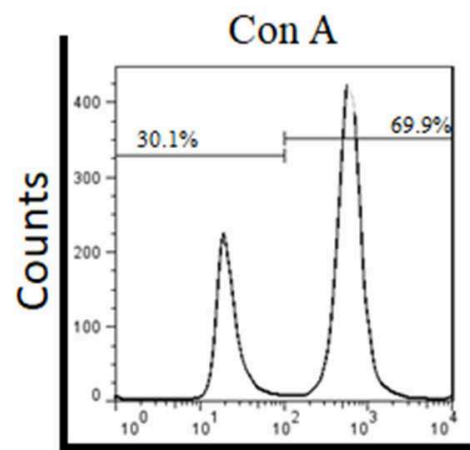

B

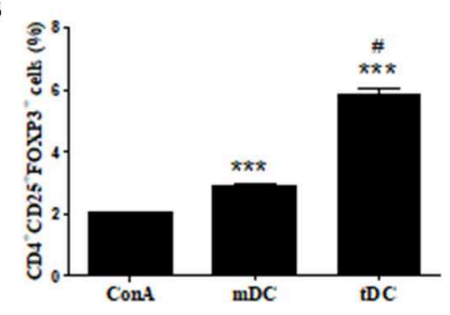

D

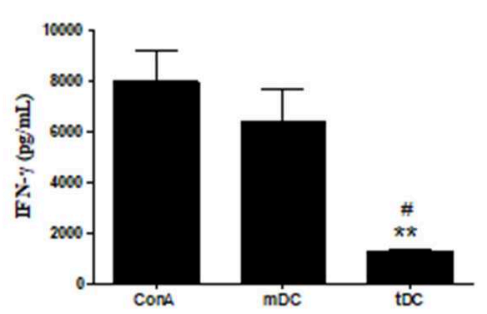

mDCs

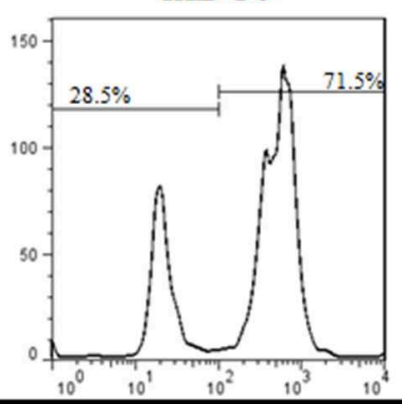

tDCs

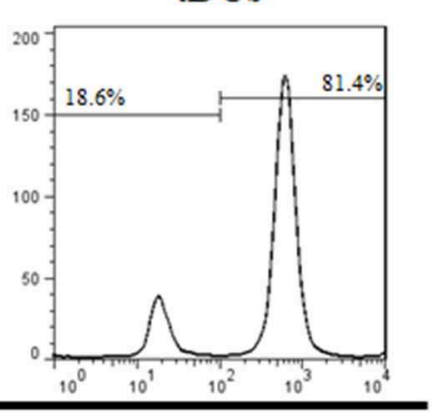

CFSE staining

FIGURE 3 | Inhibition of lymphocyte proliferation and stimulation of FoxP3 ${ }^{+}$Treg cells. (A) Effect of mDCs or tDCs on ConA-induced lymphoproliferation for 48 h. (B) Effect of $\mathrm{mDCs}$ or tDCs in induction of T regulatory (Treg) cells. (C,D) Concentrations of IL-2 and IFN- $\gamma$ determined by ELISA. (E) Effect of mDCs or tDCs on Con-A induced lymphoproliferation evaluated by CFSE staining. Values represent the mean of 9 or 12 determinations \pm SEM of three experiments performed. ${ }^{\star \star \star} P<0.001$; compared to Con A group. ${ }^{\star \star} P<0.01$; compared to Con A group. ${ }^{\#} P<0.001$; compared to mDC group.

while presenting increased production of IL-10. Altogether, these are characteristics compatible with an immunosuppressive profile $(39,40)$.
The reduction of IL-12 is highly related to the increase of IL10 production (36). This is a characteristic profile of tolerogenic dendritic cells generated from dexamethasone, which may also 

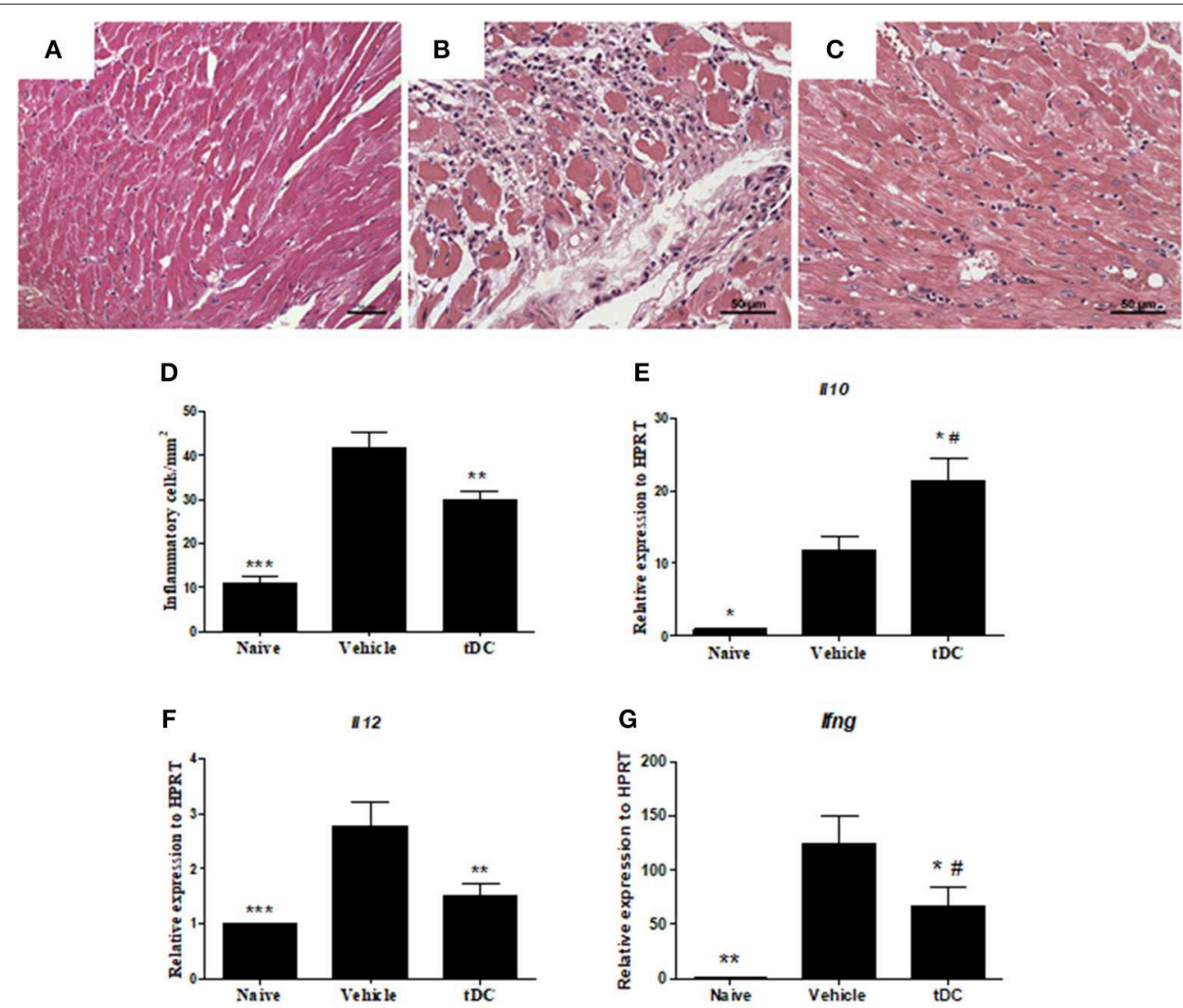

FIGURE 4 | Reduction of inflammation and quantification of the gene expression of pro and anti-inflammatory cytokines in the hearts of tDCs-treated mice. (A-C) Micrographs of hematoxylin and eosin-stained heart sections of naïve (A), vehicle-treated (B) and tolerogenic dendritic cells-treated (C) groups. (D) The number of inflammatory cells was quantified by morphometrical analysis. Gene expression of anti-inflammatory IL-10 (E) and proinflammatory IL-12 (F) and IFN $\gamma$ (G) cytokines were assessed by RT-qPCR using cDNA samples prepared from mRNA extracted from mouse hearts from the experimental groups. Values represent the mean of at least 16 determinations \pm SEM of two experiments performed with 8-10 mice/group ${ }^{\star \star \star} P<0.001$; compared to vehicle-treated mice. ${ }^{\star \star} P<0.01$; compared to vehicle-treated mice. ${ }^{\star} P<0.05$; compared to vehicle-treated mice ${ }^{\#} P<0.05$; compared to naïve mice.

have the ability to induce Treg cells $(35,36)$. The immune tolerance naturally can be achieved by different mechanisms, including $\mathrm{T}$ cell anergy, the elimination of self-reactive $\mathrm{T}$ cells or the induction of Treg cells $(36,40)$. Our results suggest that the tolerance induction by tDCs tested here occurs by induction of Treg cells belonging to the subset $\mathrm{CD} 4{ }^{+} \mathrm{CD} 25^{+}$expressing FoxP $^{+}$. The expression of FoxP $3^{+}$and differentiation of Treg cells occurs only in the presence of DC or IL-10, characterizing positive feedback regulations $(24,36,41-43)$.

Importantly, the tDCs significantly reduced the inflammatory infiltrate in the hearts of mice with CCC. This effect is probably caused by the immunosuppressive profile of tolerogenic dendritic cells, which have low immunostimulatory capacity, but with potential of inducing Treg cells via IL-10 secretion. As shown by recent studies, tolerogenic dendritic cells producing IL10 induce tolerance via activation of Treg cells in murine models of autoimmune disease $(22,34,35)$. Interestingly, in the absence of DCs, IL-10 is a cytokine which alone can induce FoxP3 expression and Treg differentiation (42). The capacity to produce IL-10 could therefore be one of the mechanisms by which tolerogenic DCs inhibit the progression of CCC, through its systemic effect, in addition to inducing the differentiation of FoxP3 ${ }^{+}$Treg cells $(25,36,41,42)$. Moreover, we found a significant reduction in IL-12 and IFN $\gamma$ gene expression in the hearts of tDCs-treated mice. The reduction of IFN $\gamma$ expression is also related to the reduction of the inflammatory infiltrate, since high levels of this inflammatory cytokine induce the activation of macrophages through the high production of IL-12 and toxic intermediates, such as nitric oxide (NO) and reactive oxygen intermediates (ROI), consequently leading to the activation of a polarized Th1 response $(44,45)$. Therefore, tDCs may also affect the maturation of DCs and macrophages, since the secretion of IL-10 interferes with the up-regulation of costimulatory molecules and inhibits the IL-12 and IFN $\gamma$ production, thus limiting the ability to promote Th1 responses (44-48).

Another important action of tolerogenic dendritic cells in the CCC was the reduction of fibrosis, which was associated with a reduction in the expression levels of colla2 and galectin 3 genes after treatment with tDCs. The role of Galectin 3 in the process of cardiac remodeling has been shown in experimental models of cardiomyopathy hypertrophy and myocardial infarction $(49,50)$. In CCC, our previous reports have shown that Gal-3 expression 

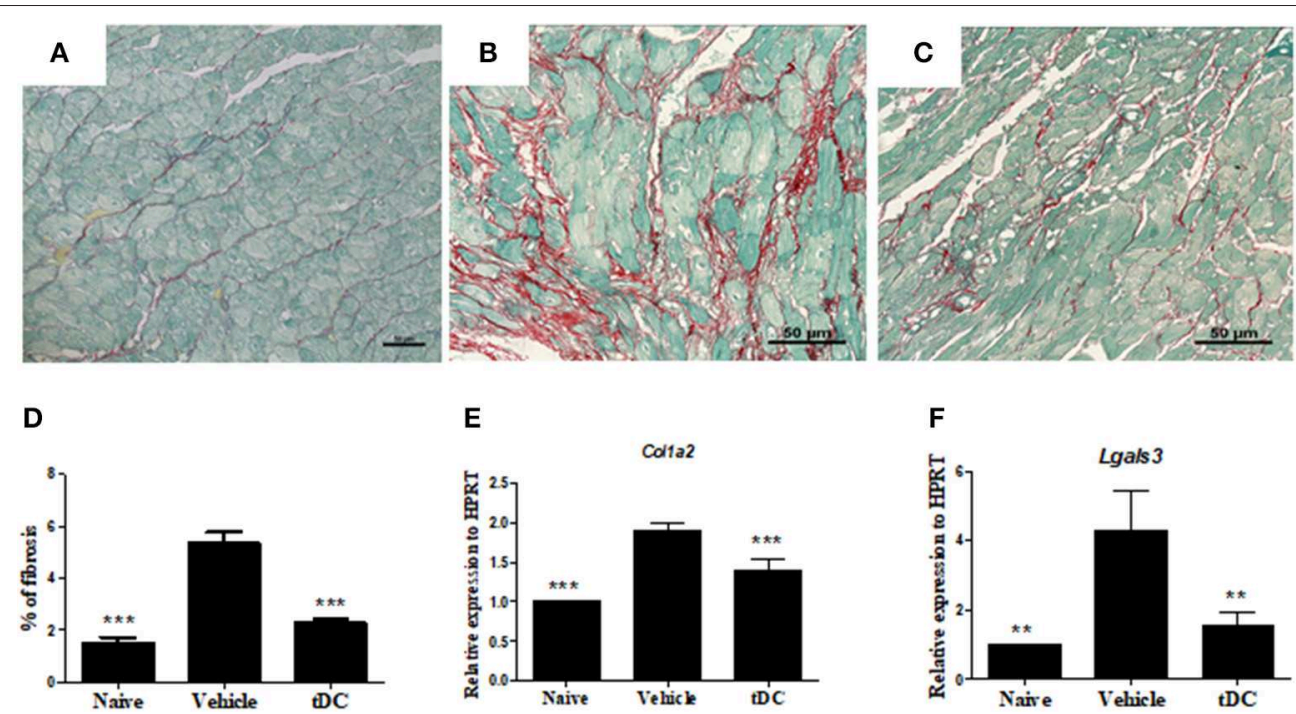

FIGURE 5 | Reduction of fibrosis and fibrosis-associated factors in the hearts of mice treated with tDCs. (A-C) Heart sections stained with Sirius-red from naïve (A), vehicle-treated (B) and tolerogenic dendritic cells-treated (C) groups. (D) The fibrosis area is represented by the percentage of collagen deposition in the heart sections. Gene expression of collagen $1 \mathrm{a} 2(\mathbf{E})$ and galectin $3 \mathbf{( F )}$ was assessed by RT-qPCR using cDNA samples prepared from mRNA extracted from hearts from experimental groups. Values represent the mean of at least 16 determinations \pm SEM of two experiments performed with $8-10$ mice/group. ${ }^{\star \star \star} P<0.001 ;$ compared to vehicle-treated mice. ${ }^{* *} P<0.01$; compared to vehicle-treated mice.

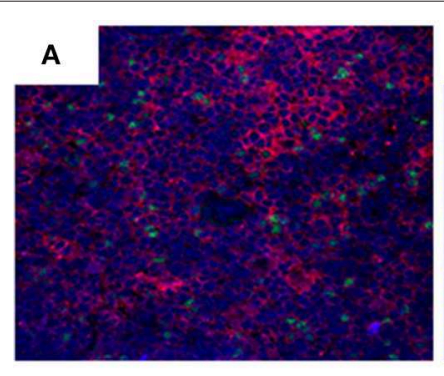

D

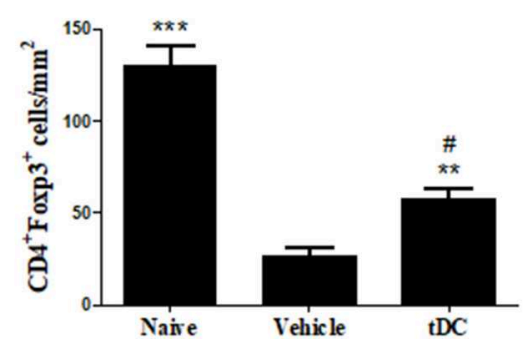

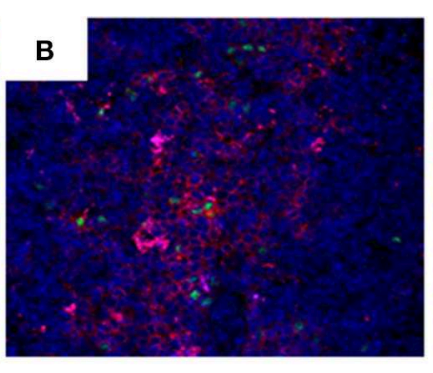

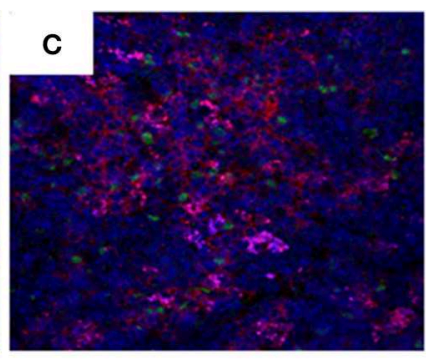

E

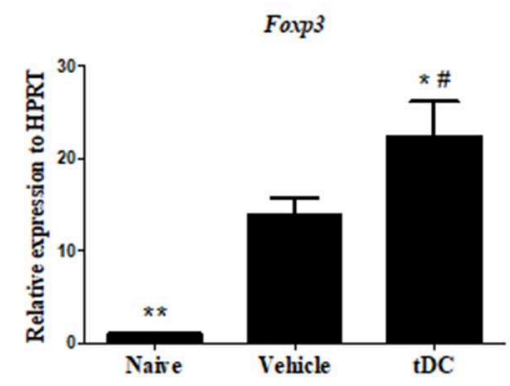

FIGURE 6 | Treatment with tDCs increases the expression of Tregs for spleen and heart. (A-C) Frozen spleen sections obtained from mice from each experimental group were stained with antibodies specific for CD4 (red), FoxP3 (green), and DAPI for nuclei staining (blue) in the spleen tissue of naïve (A), vehicle-treated (B) and tDCs-treated (C) mice. (D) Quantification of spleen sections stained for CD4 ${ }^{+}$and FoxP3 ${ }^{+}$. DAPI, 4', 6-Diamidino-2-phenylindole. The gene expression of FoxP3 (E) was evaluated by RT-qPCR using cDNA samples prepared from mRNA extracted from hearts from experimental groups. Values represent the mean of at least 16 determinations \pm SEM of two experiments performed with 8-10 mice/group. ${ }^{\star \star \star} P<0.001$; compared to vehicle-treated mice. ${ }^{\star \star} P<0.01$; compared to vehicle-treated mice. ${ }^{\star} P<0.05$; compared to vehicle-treated mice $\# P<0.001$; compared to naïve mice.

correlates with the degree of inflammation and fibrosis in the heart $(12,13,51)$. Moreover, the pharmacological blockage of this protein caused reduction of cardiac inflammation and fibrosis in infected mice (52). Thus, the reduction of fibrosis and collagen production observed after therapy with tDCs may be due to the downmodulation on galectin 3 expression.

Ours results show that tolerogenic dendritic cells have therapeutic effect on CCC, inhibiting disease progression. 
Positive effects were observed in the absence of specific antigen-pulsed tolerogenic dendritic cells. Immunotherapy with tolerogenic dendritic cells increased the levels of FoxP3 ${ }^{+}$Treg cells in the heart and spleen, implying that Treg cells promote immune modulation leading to inhibition of CCC progression.

In conclusion, this study is the first to show that treatment with bone marrow-derived tolerogenic dendritic cells effectively improves myocardial inflammation and fibrosis in murine model of chronic chagasic cardiomyopathy. This suggests the use of tolerogenic dendritic cells as a promising therapeutic strategy for treating CCC.

\section{DATA AVAILABILITY STATEMENT}

The raw data supporting the conclusions of this article will be made available by the authors, without undue reservation, to any qualified researcher.

\section{REFERENCES}

1. World Health Organization. Chagas Disease (American Trypanosomiasis). World Health Organization. Available online at: http://www.who.int/ mediacentre/factsheets/fs340/en/ (accessed January 10, 2018).

2. Coura JR. The main sceneries of chagas disease transmission. The vectors, blood and oral transmissions - A comprehensive review. Mem Inst Oswaldo Cruz. (2015) 110:277-82. doi: 10.1590/0074-0276140362

3. Coura JR, Dias JCP. Epidemiology, control and surveillance of Chagas disease: 100 years after its discovery. Mem Inst Oswaldo Cruz. (2009) 104:3140. doi: 10.1590/S0074-02762009000900006

4. Cunha-Neto E, Chevillard C. Chagas disease cardiomyopathy: immunopathology and genetics. Mediators Inflamm. (2014) 2014:683230. doi: 10.1155/2014/683230

5. Marin-neto JA, Simões MV, Junior AR. Pathogenesis of chronic Chagas cardiomyopathy : the role of coronary microvascular derangements. Rev Soc Bras Med Trop. (2013) 46:536-41. doi: 10.1590/0037-8682-00282013

6. Gutierrez FRS, Mariano FS, Oliveira CJF, Pavanelli WR, Guedes PMM, Silva GK, et al. Regulation of Trypanosoma cruzi-induced myocarditis by programmed death cell receptor 1. Infect Immun. (2011) 79:187381. doi: 10.1128/IAI.01047-10

7. Andrade JP, Marin-Neto JA, Paola AA, Vilas-Boas F, Oliveira GM, Bacal FBE. I Latin American guideline for the diagnosis and treatment of Chagas 'Heart Disease. Arq Bras Cardiol. (2011) 96:434-42. doi: 10.1590/s0066-782x2011000600002

8. Rassi A Jr, Marin-neto JA, Rassi A. Chronic Chagas cardiomyopathy : a review of the main pathogenic mechanisms and the efficacy of aetiological treatment following the BENznidazole Evaluation for Interrupting Trypanosomiasis (BENEFIT) trial. Mem Inst Oswaldo Cruz. (2017) 112:22435.doi: 10.1590/0074-02760160334

9. Blanche C, Aleksic I, Takkenberg J, Czer L, Fishbein MAT. Heart transplantation for Chagas Cardiomyopathy. Ann Thorac Surg. (1995) 60:1406-9. doi: 10.1016/0003-4975(95)00726-2

10. Silva DN, Solano B, Souza DF, Azevedo CM, Vasconcelos JF, Carvalho $\mathrm{RH}$, et al. Intramyocardial transplantation of cardiac mesenchymal stem cells reduces myocarditis in a model of chronic Chagas disease cardiomyopathy. Stem Cell Res Ther. (2014) 5:81. doi: 10.1186/ scrt470

11. Silva D, Souza B, Vasconcelos JF, Azevedo CM, Valim CX, Paredes BD, et al. Granulocyte-Colony stimulating factor-overexpressing mesenchymal stem cells exhibit enhanced immunomodulatory actions through the recruitment of suppressor cells in experimental Chagas Disease Cardiomyopathy. Fontiers Immunol. (2018) 9:1-12. doi: 10.3389/fimmu.2018. 01449

\section{ETHICS STATEMENT}

The animal study was reviewed and approved by Animal Ethics Commission of Gonndations of Ethical Issues Guideli (Approved number: 017/2017).

\section{AUTHOR CONTRIBUTIONS}

ES, LA-F, CM, JC, JV, and CN performed the experiments. ES, LA-F, CM, LP-C, and MS analyzed the data. ES, LA-F, LP-C, and MS conceived the study and wrote the manuscript.

\section{FUNDING}

This work was supported by grants from FIOCRUZ and CNPq/PROEP (grant number 400910/2013-6).

12. Vasconcelos JF, Souza BSF, Lins TFS, Garcia LMS, Kaneto CM, Sampaio GP, et al. Administration of granulocyte colony-stimulating factor induces immunomodulation, recruitment of $\mathrm{T}$ regulatory cells, reduction of myocarditis and decrease of parasite load in a mouse model of chronic Chagas disease cardiomyopathy. FASEB J. (2013) 27:4691-702. doi: 10.1096/fj.13-229351

13. Vasconcelos JF, Meira CS, Silva DN, Nonaka CKV, Daltro PS, MacAmbira SG, et al. Therapeutic effects of sphingosine kinase inhibitor N,Ndimethylsphingosine (DMS) in experimental chronic Chagas disease cardiomyopathy. Sci Rep. (2017) 7:1-14. doi: 10.1038/s41598-017-06275-z

14. Souza BSDF, Silva KN, Silva DN, Rocha VPC, Paredes BD, Azevedo $\mathrm{CM}$, et al. Galectin-3 knockdown impairs survival, migration, and immunomodulatory actions of mesenchymal stromal cells in a mouse model of Chagas Disease Cardiomyopathy. Stem Cells Int. (2017) 2017:3282656. doi: 10.1155/2017/3282656

15. Stagliano KER, Oppenheim JJ. DEXterity of tolerogenic APCs. Eur J Immunol. (2013) 43:38-41. doi: 10.1002/eji.201243184

16. Broichhausen C, Riquelme P, Geissler EK, Hutchinson JA. Regulatory macrophages as therapeutic targets and therapeutic agents in solid organ transplantation. Cotransplantation. (2012) 17:332-42. doi: 10.1097/MOT.0b013e328355a979

17. Jansen MAA, Spiering R, Broere F, van Laar JM, Isaacs JD, van Eden W, et al. Targeting of tolerogenic dendritic cells towards heat-shock proteins: a novel therapeutic strategy for autoimmune diseases? Immunology. (2017) 153:51-9. doi: 10.1111/imm.12811

18. Domogalla MP, Rostan PV, Raker VK, Steinbrink K. Tolerance through education: how tolerogenic dendritic cells shape immunity. Front Immunol. (2017) 8:1-14. doi: 10.3389/fimmu.2017.01764

19. Moreau A, Alliot-Licht B, Cuturi MC, Blancho G. Tolerogenic dendritic cell therapy in organ transplantation. Transpl Int. (2017) 30:754-64. doi: 10.1111/tri.12889

20. Huang C, Zhang L, Ling F, Wen S, Luo Y, Liu H, et al. Effect of immune tolerance induced by immature dendritic cells and CTLA4-Ig on systemic lupus erythematosus: an in vivo study. Exp Ther Med. (2017) 15:2499506. doi: 10.3892/etm.2018.5697

21. Hermansson A, Johansson DK, Ketelhuth DFJ, Andersson J, Zhou X, Hansson GK. Immunotherapy with tolerogenic apolipoprotein B-100-loaded dendritic cells attenuates atherosclerosis in hypercholesterolemic mice. Circulation. (2011) 123:1083-91.doi: 10.1161/circulationaha.110.973222

22. Aragão-França L, Rocha VJ, Cronemberger-Andrade A, Costa FHB, Vasconcelos JF, Athanazio DA, et al. Tolerogenic dendritic cells reduce airway inflammation in a model of dust mite triggered allergic inflammation. Allergy Asthma Immunol Res. (2018) 10:406-19. doi: 10.4168/aair.2018.10.4.406

23. Bell GM, Anderson AE, Diboll J, Reece R, Eltherington $\mathrm{O}$, Harry RA, et al. Autologous tolerogenic dendritic cells for 
rheumatoid and inflammatory arthritis. Ann Rheum Dis. (2017) 76:227-34. doi: 10.1136/annrheumdis-2015-208456

24. Lee JH, Kim TH, Park HE, Lee EG, Jung NC, Song JY, et al. Myosin-primed tolerogenic dendritic cells ameliorate experimental autoimmune myocarditis. Cardiovasc Res. (2014) 101:203-10. doi: 10.1093/cvr/cvt246

25. Choo EH, Lee JH, Park EH, Park HE, Jung NC, Kim TH, et al. Infarcted myocardium-primed dendritic cells improve remodeling and cardiac function after myocardial infarction by modulating the regulatory $\mathrm{T}$ cell and macrophage polarization. Circulation. (2017) 135:1444-57. doi: 10.1161/CIRCULATIONAHA.116.023106

26. Jung N, Jung $H$, Kang $M$, Lee J, Song J, Geuk H, et al. Photodynamic therapy-mediated DC immunotherapy is highly effective for the inhibition of established solid tumors. Cancer Lett. (2012) 324:58-65. doi: 10.1016/j.canlet.2012.04.024

27. Glimm H, Eaves CJ. Direct evidence for multiple self-renewal divisions of human in vivo repopulating hematopoietic cells in short-term culture. Blood. (1999) 94:2161-8. doi: 10.1182/blood.V94.7.2161.419k32_2161_2168

28. Schmittgen TD, Livak KJ. Analyzing real-time PCR data by the comparative C T method. Nat Protoc. (2008) 3:1101-8. doi: 10.1038/nprot. 2008.73

29. Rassi A Jr, Rassi A, Marin-Neto JA. Chagas disease. Lancet. (2010) 375:1388402. doi: 10.1016/S0140-6736(10)60061-X

30. Simões MV, Moreira M, Romano D, Schmidt A, Suely K, Martins M, et al. Chagas Disease cardiomyopathy. Int J Cardiovasc Sci. (2018) 31:17389. doi: 10.5935/2359-4802.20180011

31. Sallusto F. Efficient presentation of soluble antigen by cultured human dendritic cells is maintained by granulocyte/macrophage colonystimulating factor plus interleukin 4 and downregulated by tumor necrosis factor alpha. J Exp Med. (1994) 179:1109-18. doi: 10.1084/jem.179.4. 1109

32. Matyszak MK, Citterio S, Rescigno M, Ricciardi P. Differential effects of corticosteroids during different stages of dendritic cell maturation. Eur J Immunol. (2000) 30:123342. doi: 10.1002/(SICI)1521-4141(200004)30:4<1233::AID-IMMU1233>3. $0 . \mathrm{CO} ; 2-\mathrm{F}$

33. Emmer P, Van der Vlag J, Adema G, Hilbrands L. Dendritic cells activated by lipopolysaccharide after dexamethasone treatment induce donorspecific allograft hyporesponsiveness. Transplantation. (2006) 10:14519. doi: 10.1097/01.tp.0000208801.51222.bd

34. Anderson AE, Swan DJ, Sayers BL, Harry RA, Patterson AM, Delwig $\mathrm{AV}$, et al. LPS activation is required for migratory activity and antigen presentation by tolerogenic dendritic cells. J Leukoc Biol. (2009) 85:24350.doi: 10.1189/jlb.0608374

35. Laurent S, Carrega P, Saverino D, Piccioli P, Camoriano M, Morabito A, et al. CTLA-4 is expressed by human monocyte-derived dendritic cells and regulates their functions. Hum Immunol. (2010) 71:93441. doi: 10.1016/j.humimm.2010.07.007

36. Lan YY, Wang Z, Raimondi G, Wu W, Colvin BL, Creus A, et al. "Alternatively activated" dendritic cells preferentially secrete IL10, expand Foxp3+CD4+ $\mathrm{T}$ cells, and induce long-term organ allograft survival in combination with CTLA4-Ig. J Immunol. (2006) 177:5868-77. doi: 10.4049/jimmunol.177.9.5868

37. Lee JH, Park CS, Jang S, Kim JW, Kim SH, Song S, et al. Tolerogenic dendritic cells are efficiently generated using minocycline and dexamethasone. Sci Rep. (2017) 7:2-11. doi: 10.1038/s41598-017-15569-1

38. Xia CQ, Peng R, Beato F, Clare-Salzler MJ. Dexamethasone induces IL-10producing monocyte-derived dendritic cells with durable immaturity. Scand J Immunol. (2005) 62:45-54.doi: 10.1111/j.1365-3083.2005.01640.x

39. Horton C, Shanmugarajah K, Fairchild PJ. Harnessing the properties of dendritic cells in the pursuit of immunological tolerance. Biomed J. (2017) 40:80-93. doi: 10.1016/j.bj.2017.01.002
40. Nikolic T, Roep BO. Regulatory multitasking of tolerogenic dendritic cells lessons taken from vitamin D3-treated tolerogenic dendritic cells. Front Immunol. (2013) 4:113. doi: 10.3389/fimmu.2013.00113

41. Stoop JN, Harry RA, Von DA, Isaacs JD, Robinson JH, Hilkens CMU. Therapeutic effect of tolerogenic dendritic cells in established collageninduced arthritis is associated with a reduction in Th17 responses. Arthritis Care Res. (2010) 62:3656-65. doi: 10.1002/art.27756

42. Boks MA, Kager-groenland JR, Haasjes MSP, Jan J, Ham SMV. IL-10generated tolerogenic dendritic cells are optimal for functional regulatory $\mathrm{T}$ cell induction - A comparative study of human clinical-applicable DC. Clin Immunol. (2012) 142:332-42. doi: 10.1016/j.clim.2011.11.011

43. Torres-aguilar H, Aguilar-ruiz SR, Munguía R, Bajaña S, Marco A, Sánchez-torres C. Tolerogenic dendritic cells generated with differente immunosuppresive cytokines induce antigen-specific anergy and regulatory properties in memory CD4+ T cells. J Immunol. (2018) 184:176575. doi: 10.4049/jimmunol.0902133

44. Mantovani A, Sica A, Sozzani S, Allavena P, Vecchi A, Locati M. The chemokine system in diverse forms of macrophage activation and polarization. Trends Immunol. (2004) 25:677-86. doi: 10.1016/j.it.2004.09.015

45. Cunha-neto E, Teixeira PC, Fonseca SG, Bilate AM, Kalil J. Autoimmunity reviews myocardial gene and protein expression profiles after autoimmune injury in Chagas ' disease cardiomyopathy. Autoimmun Rev. (2011) 10:1635. doi: 10.1016/j.autrev.2010.09.019

46. Corinti S, Albanesi C, la Sala A, Pastore S, Girolomoni G. Regulatory activity of autocrine IL-10 on dendritic cell functions. J Immunol. (2001) 166:43128. doi: 10.4049/jimmunol.166.7.4312

47. De Smedt T, Van MM, De Becker G, Urbain J, Leo O, Moser M. Effect of interleukin-10 on dendritic cell maturation and function. Eur J Immunol. (1997) 27:1229-35. doi: 10.1002/eji.1830270526

48. Elkord E, Williams PE, Kynaston H, Rowbottom AW. Human monocyte isolation methods influence cytokine production from in vitro generated dendritic cells. Immunology. (2005) 114:204-12. doi: 10.1111/j.1365-2567.2004.02076.x

49. González GE, Cassaglia P, Noli S, Fernández MM, Wilensky L, Volberg $\mathrm{V}$, et al. Galectin-3 is essential for early wound healing and ventricular remodeling after myocardial infarction in mice. Int J Cardiol. (2014) 176:1423-5. doi: 10.1016/j.ijcard.2014.08.011

50. Yu L, Ruifrok WPT, Meissner M, Bos EM, Goor HV, Sanjabi B, et al. Genetic and pharmacological inhibition of galectin-3 prevents cardiac remodeling by interfering with myocardial fibrogenesis. Circ Heart Fail. (2013) 6:10717. doi: 10.1161/CIRCHEARTFAILURE.112.971168

51. Soares MBP, Lima RS, Souza BSF, Vasconcelos JF, Rocha LL, Ribeiro R, et al. Reversion of gene expression alterations in hearts of mice with chronic chagasic cardiomyopathy after transplantation of bone marrow cells. Cell Cycle. (2011) 10:1448-55. doi: 10.4161/cc.10.9.15487

52. Souza BSF, Silva DN, Carvalho RH, Sampaio G.LA, Paredes BD, Aragão França L, et al. Association of cardiac galectin-3 expression, myocarditis, and fibrosis in chronic Chagas Disease cardiomyopathy. Am J Pathol. (2017) 187:1134-46. doi: 10.1016/j.ajpath.2017.01.016

Conflict of Interest: The authors declare that the research was conducted in the absence of any commercial or financial relationships that could be construed as a potential conflict of interest.

Copyright (C) 2020 Santos, Aragão-França, Meira, Cerqueira, Vasconcelos, Nonaka, Pontes-de-Carvalho and Soares. This is an open-access article distributed under the terms of the Creative Commons Attribution License (CC BY). The use, distribution or reproduction in other forums is permitted, provided the original author(s) and the copyright owner(s) are credited and that the original publication in this journal is cited, in accordance with accepted academic practice. No use, distribution or reproduction is permitted which does not comply with these terms. 